\title{
Challenging the Principle of Compositionality in Interpreting Natural Language Texts
}

Françoise Gayral, Daniel Kayser and François Lévy

François Lévy, University Paris Nord, Av. J. B. Clément, 93430 Villetaneuse, France fl@ lipn.univ-paris13.fr

\section{Introduction}

The main assumption of many contemporary semantic theories, from Montague grammars to the most recent papers in journals of linguistics semantics, is and remains the principle of compositionality. This principle is most commonly stated as:

The meaning of a complex expression is determined by its structure and the meanings of its constituents.

It is also adopted by more computation-oriented traditions (Artificial Intelligence or Natural Language Processing - henceforth, NLP). Although its adequacy to "real" language clearly raises several difficulties, it is almost never challenged and the presuppositions on which it relies are seldom questioned. Rather than proposing yet more arrangements to overcome some of its drawbacks, the point of this paper is to make a more radical critique which addresses first the very notion of meaning. For us, a semantic theory should focus not on finding "the meaning of a complex expression" but on drawing appropriate inferences. This shift of focus makes it possible to adopt an approach which fulfills the tasks that the notion of meaning is supposed to fulfill, without an explicit (and compositional) construction of the meaning of parts and wholes.

The paper is organized as follows. The first part explains the reasons why compositionality has been so widely adopted and compares and contrasts what compositionality presupposes about natural language semantics with our own conception of language and semantics. The second part describes in more details and through many examples, the problems raised by the compositional hypothesis. The last part suggests a new framework which could open the way for an alternative approach. 


\section{Why has the compositional hypothesis been so widely adopted?}

The principle of compositionality offers many advantages.

- First, it can explain our ability to understand sentences we have never heard before. In the literature (Fodor \& Pylyshyn, 1988), this ability is considered from two angles:

- productivity: human beings can produce/understand infinitely many new complex sentences from finite syntactic and semantic resources;

o systematicity: each word makes a uniform contribution to the meaning of the infinite number of sentences in which it may occur.

In other words, once we have understood some complex expressions, we are able to understand others that are obtained by combining the same constituents in a different way: although the sentence is new, we are able to work out its meaning because we already know the meaning of its parts (preserved through recombination), and we also know the rule to put all those meanings together in order to get the meaning of the whole sentence.

- Second, and from a practical point of view, the compositionality principle offers a simple way to compute the meaning of complex linguistic expressions. It is one of the easiest ones that one could imagine: starting from the meanings of its constituents and following its syntactic structure, the building of the meaning of the complex expression proceeds step by step, incrementaly, from the most elementary constituents to the most complex ones.

- Third, the assumption that natural language semantics is entirely determined by syntax and lexical semantics, and that the construction of meaning is an incremental process, entails a modular vision of the interpretation process. Modularity therefore becomes the basis of the architecture of most NLP systems, so that it is possible to clearly separate a (syntactico)semantic module from a (pragmatic or inferential) module which integrates world knowledge and the elements about the situation of utterance. The first module, which has to translate natural language expressions into some suitable language (a formula of some logical calculus, or feature structures, or whatever, depending on the underlying theory), is directly concerned with compositionality and concentrates all the efforts. The second module, often considered as less linguistic in nature, is also less connected with the issue of compositionality. As it is not described as formally as the first module, it is often considered of less scientific value and thus postponed to a later phase. 


\section{Some presuppositions of compositionality}

The basic terms of the compositional hypothesis (meaning, structure), as we expressed it at the begining of this paper, are supposed to refer to clear-cut and unproblematic categories. They hide in fact at least three presuppositions.

- The first presupposition concerns the issue of meaning. The words which compose an expression, since they are the building blocks of the construction, are supposed to come with self-contained predetermined meanings ; if compositionality is assumed in its strongest sense, this meaning must be independent, both of the other words that compose the expression, and of the situation in which the sentence is uttered. A weaker form would assert that the meaning of the whole is determined by the meanings of the parts when they are fixed, but such a statement is circular, since it allows the final meaning of the parts also to depend on the meaning of the whole.

- The second presupposition concerns the notion of constituent and structure and is clearly related to syntax. Syntax embodies both the decomposition of constituents and the structure of complex expressions. These are supposed to be the ingredients in the compositional process. Hence two hypotheses are needed. First, decomposition and structure are independent of the resulting meaning (otherwise we would be trapped in a vicious circle). Second, a given syntactic structure is always involved in the same way in the elaboration of the meaning of the corresponding complex expressions. Thus, a compositional analysis generally assumes the coupling of every single syntactic rule with a semantic rule. The latter assigns a meaning to the structure built by the former, so long as it is provided with the meaning of its input, i.e. of the constituents on which it operates.

- The third one is about the process which constructs the meaning of an expression: note that at the formal level (mathematical for instance), a determined relation does not need to be algorithmically constructible. Nevertheless, compositional theories consider that determination is constructive, i.e. they consider that there exists an incremental determination process, running stepwise from the most elementary constituents to the most complex ones, composing at each step the meanings of the sub-expressions (obtained at the previous step) and following the syntactic rule underlying the constitution of the actual node.

It should be observed that this common ground commits neither to a specific conception of meaning, nor to a specific approach to syntax, as long as the necessity of the notion of meaning is not challenged. It consists just of principles that are applicable to whatever semantic theory one chooses in order to assign 
meanings to expressions of a language: Montague grammar (Montague, 1974), DRT (Kamp \& Reyle, 1993), etc. So, theories adopting the compositional hypothesis can still diverge on several points :

- the way in which syntactic and semantic analysis is done; either the syntactic analysis is considered as operating first, and as providing its output prior to any semantic analysis; or the syntactic and semantic analyses are combined as in HPSG (Pollard \& Sag, 1994): the syntactic rules integrate the semantic information obtained from the elements which are composed using this rule.

- the form of the semantic output: a logical formula in first order logic, a lambda expression, a typed-feature representation, etc.

Beyond these differences, they all agree that the process of interpretation results in attaching to each expression of a text its 'correct' meaning, and builds a (possibly under-determined) single end product. The result of the semantic step would provide an adequate representation of 'the' meaning of the text which, in turn, can either be interpreted in the appropriate models within theories where the notion of truth plays a central role and/or can serve as input to a pragmatic module that may inflect the interpretation, depending on extra-linguistic parameters.

\section{Our conception of semantics}

For us, the compositional hypothesis and the presuppositions noted above are strongly related to the generative point of view on language, where emphasis is given to the innate human ability to speak and understand, and where this ability is viewed as completely disconnected from the surrounding world. In our opinion, language should primarily be seen as a flexible means of communication. Therefore, we see no point in maintaining a tight separation between linguistic factors (morpho-syntax and semantics) and the environment in which linguistic productions are uttered (pragmatics). On the contrary, we take all dimensions of language as being equal contributors.

In more concrete terms we are trying to emancipate ourselves from the influence of the currently most popular semantic theories, and so we think necessary to ask, in naive terms, the prior question: what exactly does interpreting a text amount to?

The simplest idea that comes to mind is the observation that when someone has understood a text $\mathrm{s} / \mathrm{he}$ is able to answer questions about it ${ }^{1}$. Drawing the

\footnotetext{
${ }^{1}$ The exercises proposed at school in order to assess whether a student has understood a text, or to check whether someone has fully understood a text written in a foreign
} 
inferences that are intended from a given text is clearly an ability shared by humans. Many psychological experiments confirm this ability when they show that inference (McKoon \& Ratcliff, 1992) is everywhere, even for the interpretation of a simple noun phrase or for the basic comprehension of a text.

On the contrary, the ability to spell out 'the' meaning of each constituent of the text is rather more difficult to obtain than a judgment of whether an assertion is trivially entailed by a text. Many experiments on corpus sense tagging ${ }^{2}$ (e.g. Véronis, 2001) show that when humans have to tag a corpus according to a list of senses provided by a common dictionary, they disagree widely in their judgments. The rate of disagreement is so high for some words that it sometimes reaches the level of chance.

On the other hand, validating some (theoretical) object as being the proper meaning of a term or of a structure requires proving that this meaning can help interpretation in every situation where this structure is uttered.

It may appear unfair to some readers to compare the task of inference, which takes place at token level, with the task of attributing meaning, which it would be better to put at type level. As the only empirically available objects are utterances, i.e. tokens, it should be no surprise that asking people questions about a text gives much more coherent results than asking them about meanings, i.e. entities which are not directly accessible.

So, it is easier to test (and possibly refute) theories that concern the interpretation process than theories of meaning. This is the reason why we put inferences at the core of semantics; moreover, this allows us to consider language in all of its interactions with the world, and to see the interpretive process as a huge play of interactions between diverse pieces of information coming from various sources that are linguistic, or related to the world and to the situation of utterance. The interpretive process cannot be seen as decomposable into clearcut and sequential operations, but as a dynamic process which reaches a sort of equilibrium after all the interactions have been accounted for. The notion of equilibrium comes from an analogy with mechanics: a body submitted to various forces (attraction, repulsion) generally stabilizes at a point where their resultant cancels out. No force can be singled out and considered in isolation in order to determine this point. Similarly, a text is submitted to a number of sometimes contradictory constraints, and its preferred interpretation(s) correspond(s) to satisfying as many of them as possible.

This analogy can be taken in a strong sense, i.e. the various constraints can be given a numerical expression, and the interpretation can be found as a minimum language, confirm this opinion: the judgment will be positive if the person correctly answers a list of questions about it.

${ }^{2}$ In corpus analysis, associating meanings to words is called word sense disambiguation or sense tagging. 
of some potential (Ploux \& Victorri, 1998). We believe that the symbolic nature of language makes the recourse to numbers a bit artificial (Kayser, 1994), and that the idea of an equilibrium can be taken more metaphorically: as said above, the constraints that weigh on the interpretation originate from various sources (lexical, syntactic, semantico-pragmatic, extra-linguistic) and can be prioritized: some of them must be given greater importance without resorting to a total order, as would be the case for numbers. Using non-monotonic formalisms, it is possible to find solutions that satisfy as many constraints as possible, the importance of which are taken into account, and these solutions play a similar role in logic, as a state of equilibrium in mechanics.

This provides us with a framework in which these interactions are modeled in a uniform fashion and which puts inference at the centre. This framework will be presented in the last section.

\section{The Problems}

This section goes back over some difficulties raised when confronting real sentences to the hypotheses underlying the compositionality principle. We will examine three of them, corresponding to the presuppositions underlined in the previous sections.

- the question of meaning, in particular the handling of polysemy as a list of meanings, and the correlative view of interpretation as a disambiguation process,

- the fact that an interpretive process should assign a unique meaning for each word of an expression, and for each expression in a sentence.

- the "localism", which forces the meaning of complex expressions to depend only on the meanings of their constituents and on their structure, and thus excludes consideration of other factors.

Although these questions are not really distinct, they are separated here for the sake of presentation.

\section{The question of polysemy}

As its starting point is to know the meaning of the words, the hypothesis of compositionality immediately faces the question of polysemy.

Formal semantics (e.g. Montague, 1974) generally ignores the question and seems to consider lexical semantics (the process by which the word meaning is obtained) and grammatical semantics (the semantic construction itself) as two 
separate problems. Lexical semantics assigns a meaning to a word directly; in the case of polysemy, a disambiguation process would be necessary but it is not made explicit. Be that as it may, emphasis is put only on grammatical semantics, i.e. on the parallel constructions of syntactic and semantic structures.

This method is only legitimate if the two problems are actually separable from each other! More precisely, the meaning of a word is taken as a formal argument, merely expressed by means of a symbol, often a typographic variant of the word itself (e.g. adding a prime: man' stands for the semantic value of the word man). Consequently, the lexicon is almost void of content (just a list of empty symbols $)^{3}$.

Polysemy is therefore seen as a kind of exception that must be got rid of, one way or another. The most popular expedient to circumvent the obstacle consists in assigning a priori to a polysemic word a fixed set of symbols, often distinguished by a number (e.g. play-1, play-2, etc.). Although frequently adopted, this solution does not make any distinction between homonymy and polysemy and leaves aside two questions on which the validity of the solution crucially depends:

- how many symbols should correspond to a given word,

- the set of symbols being given, how to make explicit the procedure enabling the correct symbol to be chosen for a given occurrence of the word.

The compositional process is supposed to operate on the "right" symbol, as if the two questions had satisfactory answers, i.e. as if the disambiguating procedure could be completely independent of the problem that composition is said to resolve.

Now, evacuating this disambiguation phase and eluding the criteria which must guide it weaken the grounds on which the theory stands. Another popular way to escape the problem is to consider as often as possible that words (except in the case of homonyms) have a single (literal) meaning and that every shift of meaning is a metonymic use, or some other trope, where tropes are to be handled separately. Labeling uniformly every non literal meaning as, say, a metonymy does not help at all in finding the actual contribution of a word to the meaning of an expression in which it occurs.

Some recent approaches tackle this question instead of evading it, and try to work with a restricted form of the listing of senses in the lexicon, thereby

\footnotetext{
${ }^{3}$ In some extensions (Partee, 1995), lexical meaning postulates are added in order to describe some semantic properties associated to lexical items. They aim at connecting formal semantics with lexical semantics and at constraining the possible models which satisfy the formula corresponding to the sentence. But the lexical information expressed in these postulates remains too rudimentary to be considered as really solving the problem.
} 
eliminating from it the meanings which can be generated through composition mechanisms (Pustejovsky, 1995; Briscoe \& Copestake, 1995; Ostler \& Atkins, 1991; Nunberg, 1995). These mechanisms, including coercion or selective binding, account for some cases of systematic polysemy by exploiting richer lexical entries, but not for all, as we will now see.

The entries associated to nouns assign, in their weakest form, a type to each meaning. Lexical entries for verbs express their selection restrictions in terms of types imposed on their arguments. A verb is considered as acting as a filter on the meanings associated to its arguments. As numerous examples have proven the filters to be too rigid, type coercion softens the constraint. But the rules governing coercion are far from obvious. According to the most popular approaches, the meaning of a constituent includes the specification of a qualia structure, which encodes form, content, agentive and telic roles. When combining lexical items, the composition mechanisms can exploit this information and select from these complex structured and typed lexical entries, the type which is appropriate to the current composition rule, even in the case where there is an apparent type mismatch. So, these approaches can handle homogeneously examples such as to begin a book/a pizza, or as a fast typist/car, etc.

Now, in order to remain at the linguistic level, these techniques force the composition process to rely on a restricted operation on meaning: type matching. This is not always possible. Let us take the example of the French verb surveiller (to watch, to look after, to invigilate) and some of the very common verbal phrases in which it occurs: surveiller la piscine, les enfants, un examen, un gâteau, sa valise (to 'watch' the swimming pool, the children, an exam, a cake, your luggage) (Gayral, Pernelle \& Saint-Dizier, 2000).

The objects of these verb phrases are very different from each other and they could hardly be seen as instances of the same type. If we try to characterize their common properties when put in relation with surveiller, we can observe that what is actually watched are processes related to the object rather than the object itself: actions performed in or around a swimming pool, actions done by a child, the cooking of a cake, the fact that your suitcase is still there, etc. The common property of the object arguments is thus the existence of a process involving them, which may lead to a negative state: a child may have an accident, your cake may burn, your luggage may be stolen.

The constraints which are at work are very difficult to characterize in a systematic way and cannot be summed up just by saying that finding an activity related to the noun would solve the problem. Quite a lot of world knowledge is involved, which can clearly not be found, either in the definition of adequate types, or in qualia. For example, the telic role of the object swimming pool would contain the predicate 'swim', but no predicate describing accidents. Even if the notion of accident is somehow associated in our mind with a swimming 
pool, this cannot be considered as purely lexical information. The situation is even worse in the case of children or luggage. The only solution is the reference to world experiences, to domain knowledge, stored, for example, in scripts (Schank \& Abelson, 1977).

\section{The problem of co-presence}

With the compositional hypothesis, the interpretation of a portion of text has to be unique, and this unique interpretation must be available for every other part of the sentence. But this postulate can be challenged too, particularly when faced with a phenomenon that we call co-presence (Gayral, Kayser \& Pernelle, 2001) which is illustrated in the following examples.

(1) J'ai déposé l'examen de mercredi prochain sur ton bureau (I put next Wednesday's exam on your desk)

(2) Les examens de la semaine dernière ont déjà été corrigés (last week's exams have already been corrected)

(3) La voiture passe au feu rouge (the car is going through the red light)

(4) Etant arrêté au feu tricolore 4 (rouge) (While waiting at the threecoloured light (red))

In (1) and (2), examen refers both to an event (because of its association with a date) and to a physical object (sheet(s) of paper), according to the verb: you can neither put nor correct events!

In (3), the expression feu rouge refers both to a location (the car moves through a location) and to a time (the event happens when the light is red); so, the preposition $a u$ introduces both a place and a time complement.

In (4), the two adjectives tricolore (three-coloured) and rouge (red) do not qualify the same facet of the meaning of $f e u$ : one is static, the other dynamic.

In systems relying on the compositional hypothesis, co-presence, although fairly frequent in natural language, either yields unsolvable contradictions or remains unseen.

¿From the various observations of this section, we conclude that the solutions discussed here (e.g. Pustejovsky's generative lexicon) are half measures. They recognize the need for more knowledge and for the complexification of the composition procedures, and they believe that in this way the compositional hypothesis can be saved. But, they want qualia to remain within "linguistic" boundaries whereas the required knowledge cannot in general be reduced to a small number of role fillers, nor can it be stored in lexical entries.

\footnotetext{
${ }^{4}$ In French, traffic light are officially known as "three-coloured lights"
} 
Even if lexical knowledge were available, which would be rich enough for this objection to be overcome, another one would still remain and will be considered now: meaning cannot be determined on the basis of the portion of text within the scope of the syntactic construction. This corresponds to our next criticism.

\section{Localism and incrementality of the interpretation process in question}

Let us briefly explain what is meant exactly by localism and incrementality. According to compositionality, the only information that is available at a given node comes from the semantic information assigned to the immediately dominated nodes, together with the syntactic information determined by the combination method. That is what we call localism.

By "incremental", we mean that the interpretation process follows a strict order, the same order in which the constituents are combined to form complex expressions, step by step in a deterministic manner. At each step, the process builds the semantic value of the current node and makes it available for further steps. A semantic value for a given expression, once acquired, cannot be changed.

The consequences are twofold. First, assigning a semantic value to a node cannot depend on factors which are exterior to the portion of sentence currently being analyzed. Second, once a meaning has been assigned, it will never vary and will remain the same regardless of what the subsequent constituents (phrases, sentences, discourse) may reveal.

The following sections show that numerous counter-examples can be produced.

\section{Cases where other parts of the sentence play a role}

Consider for example the case of a simple sentence of the form NP1 V NP2 (a transitive verb V with subject NP1 and object NP2). The incremental process is generally supposed to start by combining the verb with its direct object, thus yielding a meaning for the verb phrase, and then to combine the subject with the verb phrase to find the meaning of the whole sentence (our argument would also hold for different orders of composition). That prevents the subject from influencing the meaning of the verb phrase. However, the contrast between (5) and (6) is striking.

(5) L'orage a coupé la route (the storm cut off the road)

(6) Ma voiture a coupé la route (my car cut across the road) 
The meaning of the verb couper (to cut) and thus of the verb phrase depends on the subject. In the first case, it means 'to obstruct' whereas in the second it means 'to drive from one side of the road to the other'. In English, the problem would not arise because of the phrasal verbs.

Another case is the influence, crossing the syntactic tree, of the subject over the meaning of the object, the meaning of the verb remaining more or less invariant. Let us consider:

(7) Le docteur impose un régime terrible (The doctor imposes a drastic diet)

(8) Le dictateur impose un régime terrible (The dictator imposes a drastic regime)

The verb phrase (V NP2) is identical in both cases, but NP2 has different meanings depending on the subject.

In other cases, even with an identical subject NP1 and an identical verb phrase, the interpretation of the whole sentence depends on a prepositional phrase, as in (9) and (10):

(9) Suite à des inondations, les gendarmes ont coupé la route (After a serious flooding, the police cut the road off) (the police cut = the police stopped the traffic)

(10) Entraînés dans une folle poursuite, les gendarmes ont coupé la route (caught up in a wild car chase, the police cut across the road) (the police cut $=$ the police drove across)

The contrast between (9) and (10) is a good illustration of the fact that sentences sharing the same form should not be interpreted in the same way. The difference cannot be postponed to a further pragmatic step: (9) has a durative aspect and take a time adverb pendant (during); by contrast, (10) is telic and cannot take this adverb. This is so because in (9), our knowledge about floods enables us to infer that the road is obstructed with water, branches or mud, and that these obstacles are not going to disappear instantly.

In (11) and (12) below, the different complements influence both the interpretation of the verb quitter and the object phrase l'école. In (11), quitter has the interpretation of a physical event, and l'école means the building. From (11), a norm-based inference would conclude that the next day, Paul will return to school. This inference seems implausible in (12), where quitter has a more abstract meaning, with a permanent consequence and l'école corresponds to the institution rather than to a specific determined school: the main inference drawn from (12) concerns a choice in Paul's life. 
(11) Paul quitta l'école par la fenêtre de derrière (Paul left the school through the back window)

(12) Paul quitta l'école à 16 ans pour devenir apprenti (Paul left school $l^{5}$ at 16 , in order to become an apprentice)

\section{Cases where several polysemic words constrain each other and ultimately yield a non-ambiguous interpretation}

The constraints at play are not one-way and no ordering in the composition of meanings can account for the equilibrium finally reached. In the following examples concerning combinations of the words examen (exam) and laisser (to leave), no single part of the sentence is sufficient to determine the semantic value either of the verb, or of its object:

(13) J'ai laissé l'examen de mathématiques sur ton bureau (I left the maths exam on your desk) (to leave = to put, exam = paper on which the wording of the exam is written)

(14) J'ai laissé l'examen de mathématiques à Paul qui est plus compétent (I left the maths exam to Paul who is more competent) (to leave = to entrust, exam $=$ task of writing the subject)

(15) J'ai laissé l'examen de mathématiques pour l'an prochain (I left the maths exam for next year) (to leave $=$ to postpone, exam $=$ whole process of evaluating students)

This co-influence leads to some sort of circularity that conflicts with the incrementality required by the compositional hypothesis.

\section{Cases where information external to the sentence itself is needed}

In the last three examples as in almost all of the previous ones, the necessary clues come both from factors present in the sentence and from a vast amount of world knowledge. The problem at hand is not related to the complexity of the sentences since even the interpretation of simple expressions encounters the same difficulty (Fabre, 1996). Let us take noun phrases, as (16-18) below, with just a variation in the noun of the prepositional phrase. The variation concerns words (virage, garage, ravin) which are a priori members of the same semantic category (spatial location); the preposition dans, which can have many values, takes in each of these cases its spatial meaning. Even so, the inference of the position of the car relative to the location indicated is very different in each case:

\footnotetext{
${ }^{5}$ French makes no grammatical difference between the institution and the place
} 
(16) la voiture dans le virage (the car in ${ }^{6}$ the bend)

(17) la voiture dans le garage (the car in the garage)

(18) la voiture dans le ravin (the car in the gully).

In (16), the car is on a road (a 2-D surface); in (17), it is enclosed in a closed volume; in (18), it is located 'inside' an open volume. These varying interpretations and the different inferences they trigger come neither from strictly lexical knowledge, nor from syntactic knowledge, but more from our experience of cars.

In the same vein, the adjectives red, green, yellow, three-coloured, etc. qualify the color of the object corresponding to the noun they are associated to (flag, cloth, car, etc.). Nevertheless, as we saw in examples (3) and (4) above, when three-coloured is applied to traffic lights, it refers to the permanent potentiality for the equipment to change to three lights, each of a different colour, consecutively. On the contrary, when green is applied to the same traffic light, it takes a temporal interpretation (time when the light is green).

This shows that the way the meaning of a qualifier affects the meaning of the noun it qualifies depends on elements which are external to the noun phrase.

An argument that is not going to be developed here, because it is too remote from the main theme of the paper, although it is relevant to the compositional hypothesis, concerns syntax itself. The hypothesis requires the knowledge of the syntactic structure of the sentence, i.e. the syntactic nature of the constituents and their respective roles (subject, object, etc.). In all the examples that have been examined here, this requirement is met, because the examples were deliberately kept simple. In sentences taken from real corpuses, the exact determination of the syntactic nature and role of each constituent may turn out to be the matter of much debate whereas the semantic interpretation of the whole sentence is indisputable.

\section{Cases where a plurality of worlds is needed}

Other examples are related neither to the polysemy of words, nor to a variable interpretation of a given syntactic pattern. An implicit hypothesis of compositionality is that reference is obtained by applying the (referential part of) meaning to the world at hand. But sentences often allude, explicitly or not, to a plurality of worlds, particularly when there is a question of time and when reference has to be considered at different moments. This phenomenon appears with the intensional interpretation of an expression denoting a function (e.g. 'president') which can refer to a specific person (the 'token' reading) or to the person who

${ }^{6}$ Literal translation from French 
carries out the function, whoever s/he may be (the 'type' reading). It appears too with the interpretation of some plural nominal expressions which require several worlds as well as relations between them.

Whereas the first phenomenon has been widely studied, less attention has been paid to the case of the plural. Let us examine the question through the following sentences which, though they present an identical plural expression in exactly the same syntactic pattern, lead to different interpretations:

(19) Depuis 3 mois, mes clients viennent de plus en plus souvent (during the last three months, my customers have been coming more and more frequently)

(20) Depuis 3 mois, mes clients viennent de plus en plus de loin (during the last three months, my customers have been coming from more and more distant places).

In (19), the expression mes clients (my customers) refers to a fixed set of elements. From (19), one can infer something like: the typical element of this set comes more frequently than he did 3 months ago.

In (20), the same expression refers to a "varying" set. Understanding it requires creating several sets that are not necessarily made up of the same people. What (20) induces is that the typical element of the current set lives in a more distant place than the typical element of an earlier set.

These two possible interpretations of plural noun phrases are frequent. So, the classical interpretation of plurals, namely that a plural noun phrase refers to a collection of individuals, is not sufficient to account for collections persisting over time, where their members change as in (20). In (Gayral, Kayser \& Lévy, 2001), we draw a distinction between two interpretations:

- a de dicto interpretation allows the collection to vary, but the way the collection is named holds at any time, as in (20),

- a de re interpretation refers to a fixed set of elements, as in (19). The way the collection is named helps define the members of the set without necessarily remaining relevant for the whole sentence as in the striking example (21):

(21) The fugitives are now in jail. (Enc, 1986)

Contrary to some linguists, we cannot consider that this double interpretation can be solved by evoking an ambiguity, either a lexical one - the words customer, fugitive, ... ${ }^{7}$, in themselves, would be ambiguous and could give rise to a de re (token) interpretation or a de dicto (type) one - or a grammatical one:

\footnotetext{
${ }^{7}$ And all nouns that can give rise to similar de re/de dicto interpretations.
} 
the plural definite determiner would be ambiguous too. Indeed, the mechanisms which trigger the de dicto interpretation are far from straightforward and rely on different knowledge which can be neither strictly enclosed within the lexicon, even if enriched with qualia, nor associated to some syntactic clue. External factors, such as the ability for the given individuals to satisfy a given property or to be involved in a given act, play a prominent role in the choice of a de dicto or a de re interpretation. Even if some markers can be exploited, we are again faced with a case where the 'correct' interpretation of a constituent cannot come 'from inside' and the de dicto or de re interpretation of a plural is thus 'clearly not compositional

\section{A Possible Solution}

Many of the drawbacks that have been mentioned so far are well known by the advocates of compositionality. Yet this does not seem to weaken their belief because, as we have seen, the compositionality principle is one of the easiest that one could imagine; it provides clear starting points for the interpretation process; it avoids the intervention of non linguistic knowledge, etc. It is reassuring since it applies the Cartesian principle of dividing difficult problems into small chunks, and thus avoids having to embrace all at the same time the lexicon, context, and world knowledge. What is more, this option allows NLP systems to be modular. But, as we have seen, it often leaves aside the pragmatic-inferential module, which is not considered to be directly concerned with language. On the contrary, we think that inference is necessary at every level of the interpretation process and that mastering a linguistic expression amounts to being able to draw a core set of inferences that fix its semantic relations to other expressions, and to the situation in which it is uttered. Contesting the existence of clear-cut distinctions between syntax, semantics, pragmatics, discourse, etc. we consider the interpretation process as a huge play of interactions between information coming from very different sources: linguistic (syntactic, semantic) and pragmatic, in both of its usual acceptations, i.e. related to the world and to the situation of utterance. According to this way of thinking, the interpretation process is no longer decomposable into sequential modules, and so we replace a compositional interpretation process, in which constraints propagate one way, by a system in which constraints can propagate both "bottom-up" (e.g. starting from the lexicon and the syntax) and "top-down" (e.g. starting from world knowledge) until one or more points of equilibrium are reached.

We need thus to build a system where all the constraints can be expressed. This system must embody a reasoning tool able to draw the adequate inferences and to cope with the different phenomena mentioned above. 


\section{A reasoning tool}

The project of computer scientists, and more specifically of Artificial Intelligence, regarding language understanding is to build a symbolic and logical system which offers an artificial language able to express the varied constraints at play. It is clear that these constraints, whatever level they come from (linguistic, world knowledge, information about the situation of utterance) are 'soft' constraints that can be broken and that can be potentially conflicting.

They can be broken within the 'academic' view of language: most, if not all, traditional grammars contain rules having exceptions, and despite the efforts of formal linguists, it is hard to give an account of a full language without resorting to the notion of exception. They are even more often broken, with little or no harm at all concerning comprehensibility, in everyday (non academic) speech and writing. This obvious fact did not particularly worry that much the advocates of strict compositionality either.

Therefore non-monotonicity (see the Special Issue of the Artificial Intelligence Journal on Non-Monotonic Logic, 1980), i.e. the logical property making it possible to handle rules with exceptions, is the first requirement for an adequate language-understanding system.

Second, as said earlier, our rules express both hard constraints and soft ones. Among the latter, the ability to order exceptions hierarchically and to define priorities is of great importance to be able to handle the phenomena described in this paper. This ability prohibits a property called semi-monotonicity, the technical definition of which cannot be given here; roughly, it means that, if the "hard" knowledge remains the same while "soft" constraints are added, the derived consequences can only increase. As has been observed (Brewka, 1991), this property is incompatible with putting priorities on the "soft" constraints. Therefore it is necessary to select a non-monotonic inference system that lacks semi-monotonicity whereas most of them are semi-monotonic.

Third, we have insisted on the fact that the interpretation process is not oneway and must account for the mutual influence between the interpretation of a word and the interpretation of its context. This co-influence leads to a form of circularity. The only possible way of overcoming this difficulty is to consider this process as the reaching of equilibrium. Technically, this corresponds to finding a solution to a fix-point equation: provided the sentence is comprehensible, the interaction between its different parts leads to a stable state making it possible to infer the interpretation of the whole sentence, from which one can hopefully derive the correct interpretation of each of its parts ${ }^{8}$.

\footnotetext{
${ }^{8}$ The system does not necessarily lead to a unique final state. Cases where several "stable states" are finally reached correspond to cases where the initial sentence is truly ambiguous.
} 
We have adopted Reiter's Default Logic (Reiter, 1980), which is one of the best candidates for these requirements.

- It is a default logic in which the equilibrium reached by a system of constraints is expressed as the solution of a fix-point equation. Each solution is an 'extension' (i.e. a set of propositions considered as derivable), and this is fully appropriate as an account of circularity.

- It is a non-monotonic inference system that lacks semi-monotonicity and can represent precedence among rules by the use of semi-normal defaults.

- The fact that the fix-point equation sometimes accepts several solutions is more an advantage than a problem as we have just seen.

We will show how most of the problems encountered by the compositional hypothesis can receive a tentative solution in the framework of a non-monotonic, non-semi-monotonic inference system.

\section{Accounting for linguistic and encyclopedic knowledge}

Using our approach, we write logical rules in order to express various constraints, possibly combining diverse levels of knowledge. The rules express regularities concerning the behavior of words, both when they interact with other words within some syntactic combination, and also relative to world knowledge and to the situation of utterance.

Concerning lexical information, our rules do not operate on the words themselves. We do suppose that words are associated to categories (semantic classes), but not in the sense of giving them types or qualia structures. As inference is central in our framework, the main purpose of a category is to factor out a common inferential behavior. This is very different from viewing categories - as in extensional semantics - as denoting sets of entities (Gayral \& Kayser 2001).

The criterion for categorization is thus inference invariance. For example, in a corpus of reports of road accidents (see below), the word feu (traffic light) shares with stop (both the signpost and the line on the ground), traffic sign, yellow line, etc. the functionality of regulating the traffic; all these words are related to a "legal framework"; their meaning is pure convention; their physical materialization should be made very visible, and so on.

So, for this corpus, we need a category RoadTrafficobject which bundles this information, encapsulates deontic knowledge about what this sign allows/forbids, and thus triggers inferences concerning whether a user does or does not respect the traffic rules associated to the sign. 
$\mathrm{Feu}$ possesses another type of property in this corpus: it is both a physical object (generally a pole standing at a crossroads) and an electrical device having the functionality of emitting coloured lights following a given temporal pattern. The property of being both an object and a functional device is shared by other words such as car, warning sign, engine, etc. These common factors lead to a category Device with three facets: the physical object, the mechanism it houses, and the device-related process which has time-dependent properties.

These categories are crafted just for a given purpose: they only correspond to groupings leading to correct inferences with respect to a specific context. They are not claimed to be "universal", nor are they context-independent.

For grammatical information, we suppose a surface syntactic analysis leading to simple "grammatical" predicates such as subject, object, etc. linking word occurrences together. Rules give a default semantic interpretation to these grammatical relations.

Concerning world knowledge, two important ways to structure knowledge are what Marvin Minsky introduced under the name of frames (Minsky, 1975) (the expression of how a concept is related to the entities that are directly relevant to it, and to its typical uses) and what Roger Schank named scripts (description of the unfolding of events it evokes). For example, the word car evokes its different parts (wheel, etc.); the person(s) who normally use(s) it (the driver(s)); the persons or goods that can be transported; its typical locations (road, garage, etc.), and many scripts related to driving (normal course of events as well as problematic ones: speed limits, traffic lights, jams, breakdowns, accidents, etc.). At any given level, pointers to other scripts are available (the script of puncture related to the concept wheel, the script of being repaired related to breakdown, etc.).

\section{Constructing the interpretation}

Our system will therefore be efficient if it is able to satisfy the three following requirements:

- To be able to write a set of rules adapted to its contexts of use. This requires determining the categories at play in these contexts; the static or dynamic binding of words into those categories; in the case of dynamic binding, the factors that determine the membership; and finally the effective writing of non-monotonic rules expressing the linguistic and extra-linguistic constraints concerning a domain.

- To be able to design a reasoning system that finds an equilibrium from the constraints which are active in a given interpretive process. At the beginning of the process, only the inference rules that have word occurrences in 
their premises are applicable; in their conclusion, they determine specific (and provisional) semantic values (categories) associated to these occurrences and semantic relations between them. At that point, inference rules using semantic values in their premises become accessible as well. And the process can go on.

- To be able to insure tractability and convergence; the play of constraints has to reach, in a tractable way, one or more final stable states which correspond to an adequate interpretation of the input.

It is clear that it is hard to satisfy these requirements in a general way. It can be done only in very restricted situations where the vocabulary, as well as the world knowledge involved, are limited.

We have been working for several years on a particular corpus of car-crash reports (Lévy, 1994). Just to give the flavor of what our system will look like, let us describe a mechanism corresponding to how we deal with one of the problems discussed above, namely co-presence.

In the following paragraphs, we assume that the reader is fairly familiar with Reiter's logic (Reiter 1980) and we abridge the original notations to make the rules more readable. $\mathrm{A}: \mathrm{B}[\mathrm{R} 1]$ is a shorthand for $\frac{A: B \wedge R 1}{B}$ which means: if $\mathrm{A}$ is true and if the conjunction $B \wedge R 1$ is consistent with what is known, then $B$ is true.

Let us now consider a word A (e.g. $f e u$ ) which can belong to two possible categories, say B (e.g. RoadTrafficobject) and C (e.g. Device). Each possibility is enforced only when it is considered as making sense in the given context. For the sake of simplicity (a full development would, step by step, carry us too far away), we represent this state of affairs by two propositions BM and CM (read: assigning A to category B, resp. C would be meaningful in the present context).

Two defaults will be a priori applicable:

$$
\text { (d1) } A \wedge B M: B \text { [R1] and (d2) } A \wedge C M: C \text { [R2], }
$$

i.e. as long as $B \wedge R 1$, resp. $C \wedge R 2$ is consistent (see below), the meaningfulness of an assignment is a sufficient argument to consider it as a legitimate choice.

Now, in most cases, considering $\mathrm{A}$ as a $\mathrm{B}$ makes the choice of $\mathrm{C}$ inappropriate and vice-versa. We could express this by a "strong" exclusion statement, e.g. $\mathrm{B} \Rightarrow \neg \mathrm{C}$, but this would forbid the possibility of co-presence, whereas this is precisely what we want to be able to have. We thus adopt a weaker form:

$$
\text { (d3) B : } \neg \text { R2 [R3] and (d4) C : } \neg \text { R1 [R3] }
$$

If we want to accept the co-presence of the two interpretations, it is possible to override the mutual inhibition between $\mathrm{B}$ and $\mathrm{C}$, by:

(d5) $\mathrm{BM} \wedge \mathrm{CM}: \neg \mathrm{R} 3[\mathrm{R} 1 \wedge \mathrm{R} 2]$. 
To sum up, let $\mathrm{D}$ be the set $\{\mathrm{d} 1, \ldots, \mathrm{d} 4\}$ and $\mathrm{D}$ ' the set $\{\mathrm{d} 1, \ldots, \mathrm{d} 5\}$ and let A represent the fact that the word feu occurred, (i)both theories $<\{\mathrm{A}\}, \mathrm{D}>$ and $<\{\mathrm{A}\}$, D' $>$ have a unique extension containing neither B nor C: this models the fact that, in the absence of any statement of meaningfulness, no interpretation for A is proposed; (ii)both theories $<\{\mathrm{A}, \mathrm{BM}\}, \mathrm{D}>$ and $<\{\mathrm{A}, \mathrm{BM}\}$, D' $>$ have a unique extension containing $B$ and not containing $C$; this models the fact that if A belonging to category $\mathrm{B}$ makes the sentence meaningful, then the only solution is to consider $\mathrm{A}$ as a $\mathrm{B}$; the situation is symmetric with $\mathrm{C}$; (iiia)theory $<\{\mathrm{A}, \mathrm{BM}, \mathrm{CM}\}, \mathrm{D}>$ has two extensions (one containing $\mathrm{B}$ but not $\mathrm{C}$, the other $\mathrm{C}$ but not $\mathrm{B})$, i.e. in the absence of an explicit permission for co-presence, if both readings are meaningful, the sentence is ambiguous; (iiib)theory $<\{\mathrm{A}$, $\mathrm{BM}, \mathrm{CM}\}$, D' > adds a third extension containing both B and C: if both assignments make sense, allowing for co-presence gives the choice between mutually exclusive readings (preserving the ambiguity), and a new reading in which the word enjoys simultaneously the features of the two categories. Furthermore, non-monotonicity takes into account the fact that the meaning of a sentence may appear later: suppose that, at the time of reading, no meaningful statement $\mathrm{BM}$ or $\mathrm{CM}$ is derived. According to (i) above, $\mathrm{A}$ is left uninterpreted; if the subsequent lines bring evidence that category B would make sense, i.e. allow the derivation of BM, the assignment of A to B becomes effective (case ii); conversely, if we are in case (ii) because of a default proof of BM, and if afterward some step of the proof is invalidated, we modify the comprehension of part of the text which, up till then, was considered as being understood.

For instance, the sentence:

\section{(22) The traffic-light is damaged.}

is meaningful with two different interpretations (case iiia): the pole supporting the traffic light is damaged, or the pole is OK but the lights are damaged. If we read later:

\section{(23) It is lying across the road.}

world knowledge makes it difficult to believe that the electrical device is concerned, but rather that the pole has fallen down. Going back to the occurrence of traffic light in (22), we can no longer interpret it as a member of the category Device, and we find ourselves in the case (ii) where only its attachment to Physicalobject makes sense.

These various possibilities hint at the adequacy of semi-normal defaults to handle defeasible category assignments, and prove this tool to be flexible enough to give a local permission or interdiction of co-presence, yielding either ambiguous readings (multiple extensions), or multiple meanings of a word as part of the same reading. 


\section{Conclusion}

We have shown that, despite the attractiveness of the layered architecture it would allow, the compositional hypothesis contains a number of defects which prevent it from accounting for the interpretation of natural language.

The main one concerns the treatment of polysemy. Even if one accepts a rough inventory of meanings limited to types or possibly enriched with qualia, nothing is said about how the choice of the adequate meaning can take place before the composition operates. To be acceptable, the 'magic' of this prior disambiguation phase must be disenchanted.

In addition, we have shown that a lot of very plain and common expressions/sentences cannot be understood without using information originating from outside of the subtree under consideration.

Now, if compositionality is disqualified, several other views, although commonly shared, must also be questioned. What is the importance of a complete syntactic analysis, if syntax is no longer considered as governing alone the building of interpretation? Is the aim of the interpretation process to label words, phrases, sentences, texts with unique meanings? And even to build a single end-product?

Trying to elaborate upon these points would take us far beyond the limits of an article. But we have given some indications for a framework which might be able to overcome the main drawbacks of the compositionality hypothesis: a non-monotonic system whose rules express various constraints coming from diverse levels of knowledge, and which reaches an equilibrium when all these constraints have been taken into account.

The solution we propose, although rough and incomplete, offers an alternative way of thinking about text interpretation.

\section{References}

Brewka, G. (1991). Cumulative default logic: in defense of nonmonotonic inference rules. Artificial Intelligence Journal, 50(2), 183-205.

Briscoe, T., \& Copestake, A. (1995). Semi-productive polysemy and sense extension. Journal of Semantics, 12(1), $1-53$.

Enc, M. (1986). Towards a referential analysis of temporal expressions. Linguistics \& Philosophy, 9, 405-426.

Fabre, C. (1996). Interprétation automatique des séquences binominales en anglais et en français. Application à la recherche d'informations. Unpublished doctoral dissertation, Université de Rennes.

Fodor, J., \& Pylyshyn, Z. (1988). Connectionism and cognitive architecture. Cognition, 28, 3-71.

Gayral, F., \& Kayser, D. (2001). Categorisation seen as factorisation of the inferential potential. Application to the comprehension of utterances. Cognitive Systems, 5(4), 345-371.

Gayral, F., Kayser, D., \& Lévy, F. (2001). Plurals, time, and world knowledge. In International conference recent advances in NLP (RANLP). Tzigov Chark, Bulgaria. 
Gayral, F., Kayser, D., \& Pernelle, N. (2001). In search of the semantic value(s) of an occurrence: an example and a framework. In H. Bunt, R. Muskens, \& E. Thijsse (Eds.), Computing meaning (Vol. 2, pp. 53-69). Kluwer.

Gayral, F., Pernelle, N., \& Saint-Dizier, P. (2000). On verb selectional restrictions: Advantages and limitations. In 2nd international conference on natural language processing (NLP) (pp. 57-68). Grèce.

Kamp, H., \& Reyle, U. (1993). From discourse to logic (Vol. II). Dordrecht: Kluwer.

Kayser, D. (1994). Continuity in linguistic semantics. In C. Fuchs \& B. Victorri (Eds.), (pp. 111-126). John Benjamins.

Lévy, F. (Ed.). (1994). Approches sémantiques. (Vol. 13, No. 1)

McKoon, G., \& Ratcliff, R. (1992). Inference during reading. Psychological review, 99(3), 440-466.

Minsky, M. (1975). A framework for representing knowledge. In P. H. Winston (Ed.), The psychology of computer vision (pp. 211-275). McGraw-Hill.

Montague, R. (1974). Formal philosophy: Selected papers of Richard Montague. New Haven: Yale University Press,.

Nunberg, G. (1995). Transfers of meaning. Journal of Semantics, 12.

Ostler, N., \& Atkins, B. (1991). Predictable meaning shift: some linguistic properties of lexical implication rules. In Springer-Verlag (Ed.), Lexical semantics and knowledge representation (pp. 88-100). Berkeley, CA.

Partee, B. (1995). Lexical semantics and compositionality. In M. L. L. Gleitman (Ed.), Language part i (pp. 311-360). Cambridge, MA: MIT Press.

Ploux, S., \& Victorri, B. (1998). Construction d'espaces sémantiques à l'aide de dictionnaires de synonymes. Traitement automatique des langues, 39(1), 161-182.

Pollard, C. J., \& Sag, I. A. (1994). Head-driven phrase structure grammar. University of Chicago Press.

Pustejovsky, J. (1995). The generative lexicon. Cambridge, MA: MIT Press.

Reiter, R. (1980). A logic for default reasoning. AI, 81-132.

Schank, R., \& Abelson, R. P. (1977). Scripts, plans, goals and understanding. Hillsdale, NJ: Lawrence Erlbaum Ass.

Special issue of the artificial intelligence journal on non-monotonic logic. (1980, April). (Vol. 13, No. 1-2)

Véronis, J. (2001). Sense tagging: does it make sense? In Corpus linguistics '2001 conference. Lancaster, U.K. 